\title{
Safety and antitumor activity of recombinant soluble Apo2 ligand
}

\author{
Avi Ashkenazi, ${ }^{1}$ Roger C. Pai, ${ }^{2}$ Sharon Fong, ${ }^{1}$ Susan Leung, ${ }^{2}$ David A. Lawrence, ${ }^{1}$ \\ Scot A. Marsters, ${ }^{1}$ Christine Blackie, ${ }^{2}$ Ling Chang, ${ }^{1}$ Amy E. McMurtrey, ${ }^{3}$ Andrea Hebert ${ }^{4}$ \\ Laura DeForge, ${ }^{4}$ Iphigenia L. Koumenis, ${ }^{2}$ Derf Lewis, ${ }^{5}$ Louise Harris, ${ }^{6}$ Jeanine Bussiere, ${ }^{6}$ \\ Hartmut Koeppen, ${ }^{7}$ Zahra Shahrokh, ${ }^{2}$ and Ralph H. Schwall ${ }^{1}$
}

${ }^{1}$ Department of Molecular Oncology,

${ }^{2}$ Department of Process Sciences,

${ }^{3}$ Department of Cell Biology and Technology,

${ }^{4}$ Department of Research Bioassays,

${ }^{5}$ Department of Analytical Chemistry,

${ }^{6}$ Department of Pharmacological Sciences, and

${ }^{7}$ Department of Pathology, Genentech Inc., South San Francisco, California 94080-4990, USA

Address correspondence to: Avi Ashkenazi, Department of Molecular Oncology, Genentech Inc., 1 DNA Way, South San Francisco, California 94080-4990, USA. Phone: (650) 225-1853; Fax: (650) 225-6443; E-mail: aa@gene.com.

Received for publication March 26, 1999, and accepted in revised form June 10, 1999.

\begin{abstract}
TNF and Fas ligand induce apoptosis in tumor cells; however, their severe toxicity toward normal tissues hampers their application to cancer therapy. Apo2 ligand (Apo2L, or TRAIL) is a related molecule that triggers tumor cell apoptosis. Apo2L mRNA is expressed in many tissues, suggesting that the ligand may be nontoxic to normal cells. To investigate Apo2L's therapeutic potential, we generated in bacteria a potently active soluble version of the native human protein. Several normal cell types were resistant in vitro to apoptosis induction by Apo2L. Repeated intravenous injections of Apo2L in nonhuman primates did not cause detectable toxicity to tissues and organs examined. Apo2L exerted cytostatic or cytotoxic effects in vitro on 32 of 39 cell lines from colon, lung, breast, kidney, brain, and skin cancer. Treatment of athymic mice with Apo2L shortly after tumor xenograft injection markedly reduced tumor incidence. Apo2L treatment of mice bearing solid tumors induced tumor cell apoptosis, suppressed tumor progression, and improved survival. Apo2L cooperated synergistically with the chemotherapeutic drugs 5-fluorouracil or CPT-11, causing substantial tumor regression or complete tumor ablation. Thus, Apo2L may have potent anticancer activity without significant toxicity toward normal tissues.

J. Clin. Invest. 104:155-162 (1999).
\end{abstract}

\section{Introduction}

Apoptosis is essential for development and homeostasis in metazoans (1). Mammals have evolved a unique apoptosis-signaling mechanism that actively instructs individual cells to die. This mechanism involves interaction of death ligands such as TNF and Fas ligand (FasL) with cell-surface death receptors such as TNF receptor 1 (TNFR1) and Fas/Apo1/CD95; these death receptors directly engage the cell's apoptotic caspase machinery ( 2 , $3)$. Instructive apoptosis plays a physiological role in deletion of activated lymphocytes at the end of an immune response and in elimination of virus-infected cells and oncogenically transformed cells.

Death ligands harbor potential as cancer-therapeutic agents because they can trigger apoptosis in many types of tumor cells. Chemotherapeutic drugs and radiation therapy usually require function of the p 53 tumor-suppressor gene for antitumor activity (4); however, more than half of human tumors acquire inactivating p53 mutations, thereby becoming resistant to therapy. Death ligands induce apoptosis independently of p53 and, thus, may offer a complementary approach to conventional cancer therapy.

Despite the ability of TNF and FasL to induce apopto- sis in cancer cells, severe toxic side effects preclude both of these ligands from use in systemic anticancer therapy. TNF infusion causes a lethal inflammatory response that resembles septic shock; this effect is mediated primarily by TNF's activation of the proinflammatory transcription factor NF- $\mathrm{KB}$ in vascular endothelial cells and macrophages (5). Infusion of agonistic anti-Fas antibody causes lethal liver damage; this damage is caused by induction of Fas-dependent apoptosis in hepatocytes, which express high levels of Fas (2).

Apo2 ligand (Apo2L, or TRAIL) was discovered because of its sequence homology to TNF and FasL (6, 7). Apo2L triggers apoptosis through interaction with the death receptors DR4 (8) and DR5 (9-15). In contrast to TNF and FasL, Apo2L mRNA is expressed constitutively in many tissues $(6,7)$, which suggests the existence of physiological mechanisms that can protect many normal cell types from induction of apoptosis specifically by Apo2L. One such mechanism may involve expression of antagonistic decoy receptors that can compete with DR4 and DR5 for ligand binding; Apo2L interacts with 3 such decoys: DcR1 (9, 10, 13, 16, 17), DcR2 (18-20), and osteoprotegerin (OPG) $(21,22)$.

Most TNF family ligands are type 2 transmembrane 
proteins; upon cleavage by specific proteases, they can form soluble homotrimeric molecules (23). Whereas soluble TNF has strong agonist activity (5), soluble FasL is a very weak agonist and can antagonize the function of membrane-associated FasL, which has potent apoptosis-inducing activity (24). Apo2L is also expressed as a type 2 transmembrane protein $(6,25,26)$, and its extracellular region forms a soluble molecule upon cleavage (25). A polyhistidine-tagged soluble form of human Apo2L (amino acids 114-281) was biologically active (6, 27). In contrast, a Flag epitope-tagged form of human Apo2L (amino acids 95-281) was poorly active and required oligomerization by anti-Flag antibody (7) for potent biological activity. Recent work describes a soluble Apo2L fusion protein (termed LZ-TRAIL) in which the extracellular region of the ligand (amino acids 95-281) is linked to an exogenous, modified leucine zipper that drives trimerization; this molecule is mostly homotrimeric and has biological activity (28). Injection of the LZ-TRAIL fusion protein in mice did not reveal any toxicity, and the molecule exhibited significant antitumor activity (28).

Here we describe a recombinant soluble form of human Apo2L that is devoid of foreign sequence (amino acids 114-281). This molecule forms stable homotrimers and has potent apoptosis-inducing activity. We report the results of studies on the safety of the soluble human Apo2L molecule in nonhuman primates. In addition, we describe studies in xenograft models that assess antitumor activity of soluble Apo2L as a single agent and in combination with chemotherapy. Our results support the hypothesis that Apo2L may prove to be safe and effective as a cancer-therapeutic agent.

\section{Methods}

Apo2L expression and purification. The extracellular portion of human Apo2L (amino acids 114-281) was subcloned into the pS1346 expression plasmid (29) with an added initiator methionine codon, and was expressed under control of the trp promoter in Escherichia coli strain W3110 in $10 \mathrm{~L}$ or $100 \mathrm{~L}$ of fermentors. Cell paste containing recombinant human soluble Apo2L was extracted with a buffer containing $0.1 \mathrm{M}$ Tris, $0.2 \mathrm{M} \mathrm{NaCl}, 50$ mM EDTA ( $\mathrm{pH} 8.0$ ). The extract was precipitated by $40 \%$

\section{Figure 1}

Oligomeric state and bioactivity of bacterially produced soluble human Apo2L. (a) Denaturing SDS-PAGE analysis of purified soluble Apo2L (2 $\mu \mathrm{g})$ without (-) or with (+) reduction by $25 \mathrm{mM}$ of DTT. Molecular weight markers are shown in the left lane. The gel was stained with silver. Identical results were obtained when reduced and nonreduced $A p o 2 L$ was run on separate gels (not shown). (b) Nondenaturing chromatographic analysis of purified soluble Apo2L. Purified Apo2L $(2 \mu \mathrm{g})$ was run on a Superose 12 size-exclusion HPLC column in a buffer containing $400 \mathrm{mM}$ ammonium sulfate and $13 \mathrm{mM}$ sodium phosphate $(\mathrm{pH} \mathrm{6.5)}$ and was detected by absorbance at $214 \mathrm{~nm}$. The elution time of molecular weight markers is indicated by arrows. The peak appearing at 29-30 minutes is from buffer elution at the included volume of the column. (c) Measurement of Apo $2 \mathrm{~L}$ concentration. Representative standard curve for Apo $2 \mathrm{~L}$ concentration as determined by ELISA with 2 monoclonal anti-human Apo2L antibodies that recognize epitopes in Apo2L's extracellular region. (d) Measurement of Apo2L bioactivity. Representative standard curve for Apo2L cytotoxicity toward SK-MES-1. ammonium sulfate. Purification to $>98 \%$ homogeneity was achieved by 2 consecutive chromatographic separation steps on hydroxyapatite and Ni-NTA agarose columns. (Although it lacks a polyhistidine tag, the recombinant soluble 114-281 Apo2L fragment bound to the Ni-NTA column, probably through endogenous histidine residues.) Purity was determined by SDS-PAGE and silver nitrate or Coomassie blue staining, by amino acid sequence analysis, and by size-exclusion HPLC.

Apo2L ELISA. Microtiter wells were coated with antiApo2L mAb 2G9, then incubated with Apo2L-containing samples followed by biotinylated anti-Apo2L mAb 2E11 (which binds to an independent epitope), and developed with streptavidin-horseradish peroxidase (Amdex; distributed through Amersham Pharmacia Biotech, Piscataway, New Jersey, USA) and tetramethyl benzidine substrate solution (Kirkegaard \& Perry Laboratories, Gaithersburg, Maryland, USA). The concentration of Apo2L in each sample was extrapolated from a 4-parameter fit of the Apo2L standard curve. Standard curves were generated with serial dilutions of purified Apo2L, the concentration of which was determined by absorbance at $280 \mathrm{~nm}$ on the basis of an extinction coefficient of 1.4 .

Apo2L bioactivity assays. SK-MES-1 human lung carcinoma cells (American Type Culture Collection, Rockville, Maryland, USA) were seeded in 96-well plates $\left(4 \times 10^{4}\right.$ per well $)$ and incubated with Apo2L for 24 hours. Cell viability was determined by crystal violet staining. Cancer cell lines developed at the US National Cancer Institute (NCI; Bethesda, Maryland, USA) were incubated with serial dilutions of Apo2L for 48 hours, and cell growth was determined by a sulforhodamine $\mathrm{B}$ protein assay as described (30). Normal human primary cells were obtained from Clonetics, San Diego, California, USA.

Study of Apo2L safety in nonhuman primates. Human Apo2L is capable of killing mouse cell lines similar to

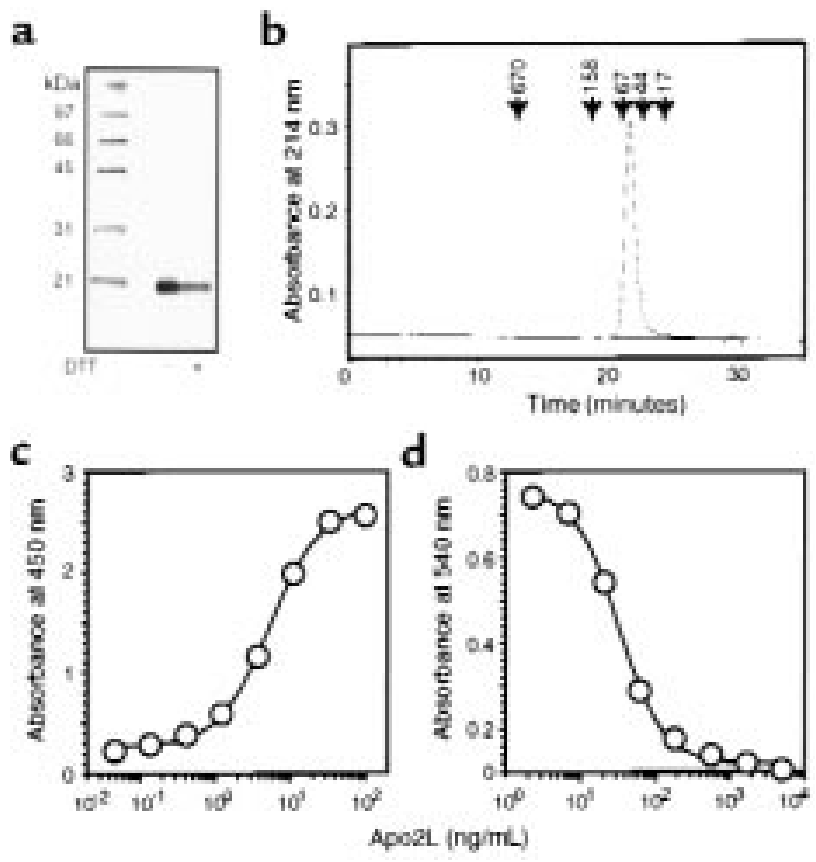




\section{Figure 2}

Safety of Apo2L. (a) Human umbilical cord endothelial cells (HUVEC) were incubated with purified soluble TNF, LT- $\alpha$, or Apo2L for 1 hour, and $\mathrm{NF}-\kappa \mathrm{B}$ activity in nuclear extracts was determined by electrophoretic mobility-shift assay (38). (b) HUVEC, normal human lung fibroblasts (NHLF), human mammary epithelial cells (HMEC), colon smooth muscle cells (COSMC), prostate epithelial cells (PREC), renal proximal tubule epithelial cells (RPTEC), or normal human astrocytes (NHA) were incubated for 24 hours with Apo $2 \mathrm{~L}(1 \mu \mathrm{g} / \mathrm{mL})$, and cell death was measured by FACS ${ }^{\circledR}$ analysis of propidium iodide staining. (c) Baboon CB1 cells were treated with vehicle or $\mathrm{Apo} 2 \mathrm{~L}$ in the presence of $10 \mu \mathrm{g} / \mathrm{mL}$ cycloheximide for 16 hours, and apoptosis was determined as in b. (d) Serum samples from cynomolgus monkeys treated with various doses of $A p o 2 L$ were analyzed for Apo2L concentration by ELISA, and for Apo2L activity by bioassay, as in Figure 1, c and d. (e) Histological sections of liver and lung tissue from cynomolgus monkeys treated with vehicle or Apo2L (10 $\mathrm{mg} / \mathrm{kg} / \mathrm{d}$ ) for 7 days. a

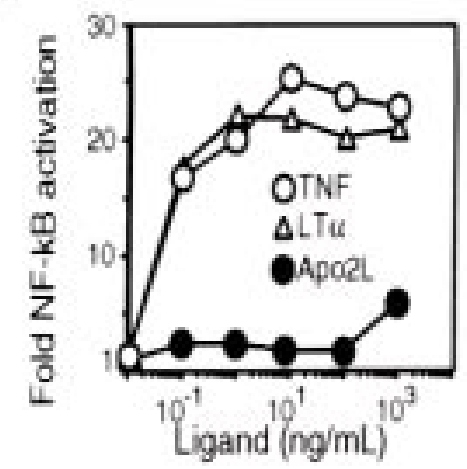

b

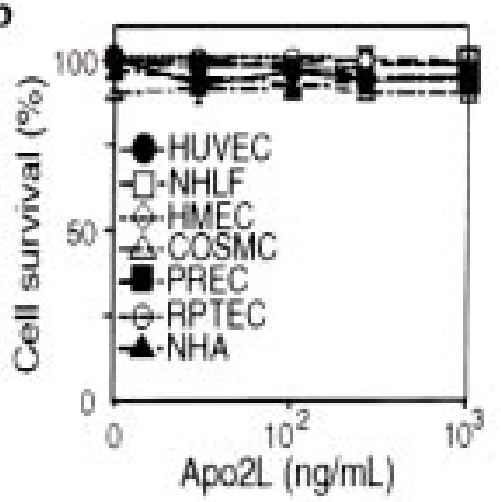

\section{c}

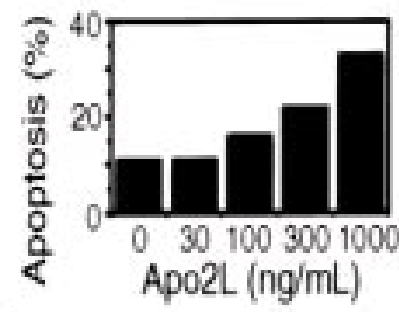

d

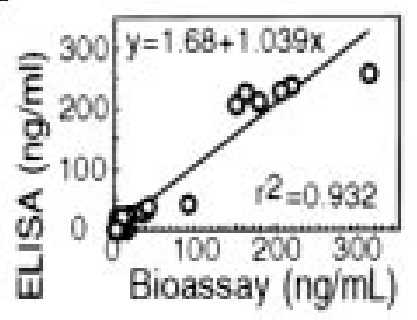

e

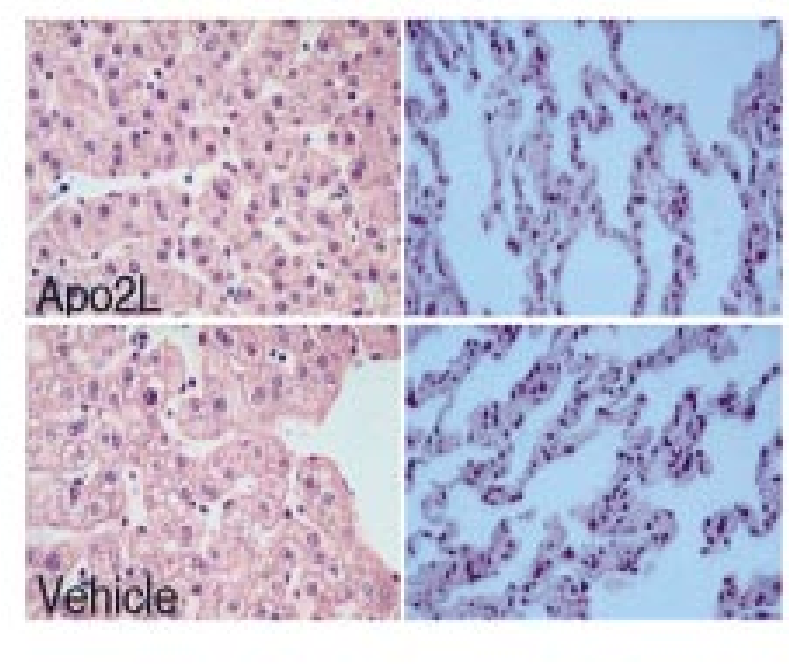

mouse Apo2L $(7,28)$. Because mice are relatively resistant to the toxicity of TNF, we chose to test the safety of Apo2L in nonhuman primates. To preserve Apo2L material, we used cynomolgus monkeys, which are relatively small. We first confirmed that human Apo2L induces apoptosis in nonhuman primate cells; because cynomolgus cell lines are not available, we used the baboon lymphoblastoid cell line CB-1 (American Type Culture Collection). Human Apo2L induced significant apoptosis in these cells (see Figure 2c). Next, cynomolgus monkeys (2 per group) received intravenous injections of recombinant soluble human Apo2L (lot 30430A) for 7 days at doses of $0,0.1,1$, or $10 \mathrm{mg} / \mathrm{kg} / \mathrm{d}$. Animals were observed for any abnormal clinical signs 1 day before dosing; at $0.5,1,2,4$, and 6 hours after dosing on day 1 ; and twice daily thereafter. Body weights were measured on day 1 before dosing, on days 2 and 7, and on day 9, before necropsy. Organ weights and histopathology were assessed on day 9. Systolic blood pressure and a veterinary physical exam were performed 1 week before dosing and at days 2 and 7 after dosing. Blood samples were collected at frequent intervals throughout the study for analysis of serum chemistry, hematology, and coagulation profiles. Urine was collected for standard urinalysis 1 week before dosing and on days 1, 2, and 7 of dosing. Serum samples collected from all study animals on day 1 before dosing and on day 9 were analyzed by ELISA for the presence of anti-Apo2L antibody titers.

In vivo analysis of antitumor activity. Athymic nude mice (The Jackson Laboratory, Bar Harbor, Maine, USA) were injected subcutaneously with $10^{6}$ HCT116, or $5 \times 10^{6}$ COLO201 or COLO205, human colon carcinoma cells (American Type Culture Collection). Apo2L was administered intraperitoneally, usually by injection, or in some experiments by implantation of osmotic infusion minipumps (Alzet 1000D; Alza Corp., Palo Alto, California, USA). Chemotherapy was given by intraperitoneal injection. 5-FU (5-fluorouracil) was purchased from Roche Laboratories (Nutley, New Jersey, USA). CPT-11 (Camptosar) was purchased from Pharmacia and Upjohn Co. (Kalamazoo, Michigan, USA).

\section{Results}

Recombinant soluble buman Apo2L forms an active homotrimer. To examine the activity of a soluble human Apo2L molecule that contains minimal heterologous sequence, and is therefore less likely to be immunogenic in humans, we constructed a bacterial expression vector encoding human Apo2L amino acids 114-281, preceded only by an initiating methionine. We expressed the protein in E. coli, extracted it from cell pastes, and purified it to essential homogeneity by chromatographic techniques (see Methods). $\mathrm{NH}_{2}$-terminal sequence analysis of the purified protein showed that the added initiating 


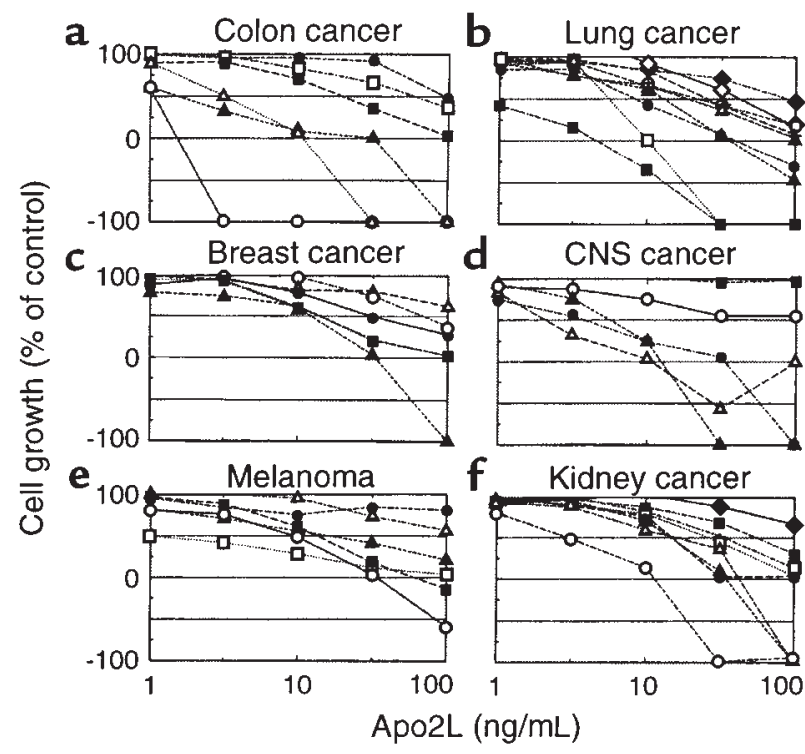

Figure 3

Sensitivity of cancer cell lines to Apo2L. Cells were incubated with seria dilutions of $\mathrm{Apo} 2 \mathrm{~L}$ for 48 hours, and cell growth relative to buffer controls was determined. (a) Colon cancer lines: COLO205 (open circles), HCT15 (open triangles), HCT116 (filled triangles), HCC-2998 (filled squares), SW620 (open squares), HT29 (filled circles). (b) Lung cancer cell lines: NCl-H460 (filled squares), HOP-92 (open squares), HOP-62 (filled triangles), $\mathrm{NCl}-\mathrm{H} 226$ (filled circles), $\mathrm{NCl}-\mathrm{H} 322 \mathrm{M}$ (open triangles), EKVX (plus signs), $\mathrm{NCl}-\mathrm{H} 522$ (open circles), A549 (open diamonds), $\mathrm{NCl}-\mathrm{H} 23$ (filled diamonds). (c) Breast cancer cell lines: MDA-MB-231 (filled triangles), MDA-N (filled squares), MCF7 (filled circles), BT-549 (open circles), NCl/ADR-RES (open triangles). (d) Central nervous system (CNS) cancer cell lines: SF-539 (filled triangles), SF-295 (open triangles), U251 (filled circles), SF-268 (open circles), SNB-75 (filled squares). (e) Melanoma cell lines: MALME-3M (open circles), M14 (filled squares), SK-MEL-28 (open squares), SK-MEL-2 (filled triangles), UACC257 (open triangles), SK-MEL-5 (filled circles). (f) Renal cancer cell lines: RXF-393 (open circles), ACHN (filled triangles), TK-10 (open triangles), A498 (filled circles), CAKI-1 (filled circles), SN12C (open squares), UO31 (filled squares), 786-0 (filled diamonds).

methionine was spontaneously removed by proteolysis in the E. coli host, thus generating a mature Apo2L polypeptide that starts at amino acid 114 of the fulllength Apo2L protein (data not shown). Denaturing gel electrophoresis of the purified protein revealed a single band of $\sim 20 \mathrm{kDa}$ (Figure 1a), which is consistent with the calculated molecular mass of $19.6 \mathrm{kDa}$ of the recombinant Apo2L polypeptide. Size-exclusion chromatography under nondenaturing conditions showed a relative molecular mass of $60 \mathrm{kDa}$ (Figure $1 \mathrm{~b}$ ), which indicates that the bacterially expressed soluble Apo2L molecule forms stable homotrimers.

As a tool for measuring Apo2L concentration, we developed an ELISA based on 2 mouse anti-human Apo2L mAb's (Figure 1c). To test Apo2L activity, we incubated human lung carcinoma SK-MES-1 cells with Apo2L and determined cell viability after 24 hours. Apo2L induced substantial cell death, with half-maximal killing at $~ 30$ $\mathrm{ng} / \mathrm{mL}(0.5 \mathrm{nM})$ (Figure 1d). Analysis of cell morphology, DNA fragmentation, and phosphatidylserine exposure confirmed that Apo2L-induced cell death occurred through apoptosis (data not shown). Thus, unlike soluble
FasL, which seems to act as an antagonist (24), soluble Apo2L forms a stable homotrimeric molecule that has potent apoptotic-agonistic activity.

Apo2L is a weak activator of NF- $K B$. To test whether Apo2L has proinflammatory activity that resembles that of TNF, we assayed its ability to activate the transcription factor NF-KB in cultured vascular endothelial cells (Figure 2a). Apo2L induced a detectable increase in NF- $\mathrm{KB}$ activity; however, TNF and lymphotoxin- $\alpha($ LT- $\alpha)$ induced a much stronger response. Furthermore, the stimulation by Apo2L required 3-4 orders of magnitude higher ligand concentrations than did stimulation by TNF or LT- $\alpha$. Similar results were obtained in human HeLa cells (data not shown). These data indicate that Apo2L is substantially weaker than TNF or LT- $\alpha$ at activating NF- $\mathrm{KB}$.

Various normal buman cell types are resistant to apoptosis induction by Apo2L. To test whether Apo2L initiates apoptosis in normal cells, we exposed early-passage primary human umbilical vein endothelial cells; lung fibroblasts; mammary, renal, or prostatic epithelial cells; colon smooth muscle cells; or astrocytes to Apo2L $(1 \mu \mathrm{g} / \mathrm{mL})$. We observed no morphological evidence of apoptosis (not shown); in addition, there was no change in staining of the cells by propidium iodide (Figure $2 \mathrm{~b}$ ), which indicated that Apo2L was not cytotoxic toward these cell types.

Safety of Apo2L in nonhuman primates. Studies in mice show that repeated injections of the LZ-TRAIL fusion protein are tolerated without obvious toxicity (28). Because rodents are much less sensitive to TNF's toxicity than are primates (31), we assessed the effect of soluble Apo2L injection in nonhuman primates. Human Apo2L is cytotoxic toward certain mouse cell lines $(7,28)$, which indicates that the human ligand has a relatively broad species cross reactivity. Consistent with this conclusion, human Apo2L was cytotoxic toward the baboon lymphoblastoid cell line CB1 (Figure 2c). To assess the safety of Apo2L in vivo, we injected cynomolgus monkeys intravenously with vehicle or with human Apo2L at doses of $0.1,1$, and $10 \mathrm{mg} / \mathrm{kg} / \mathrm{d}$ for 7 days. The specific bioactivity of Apo2L in the serum of treated monkeys, as measured in the SK-MES- 1 cell bioassay, showed a strong correlation $\left(r^{2}=0.932\right)$ to the serum concentration of Apo2L, as measured by ELISA (Figure 2d), indicating that the protein remained active while in the blood circulation. We subjected the animals to a comprehensive set of clinical tests, before and after the treatment period. Apo2L did not affect body weight, body temperature, blood pressure, or heart rate. All the animals, including the controls, showed some evidence of mild anemia at day 9, with small decreases in hemoglobin, red blood cells, and hematocrit; this effect was most likely due to frequent blood drawing during the study. There were no Apo2L-associated changes in leukocyte counts, liverenzyme activity, coagulation profiles, serum chemistry, or urine analysis. Histological evaluation of liver, lung (Figure 2e), heart, brain, kidney, ovary, testis, spleen, bone marrow, and lymph nodes (data not shown) did not reveal Apo2Lrelated adverse changes. In particular, there was no morphological evidence of hepatotoxicity or lymphotoxicity, and bone marrow sections displayed normal cellularity and progressive maturation of all lineages. Analysis of day 7 serum samples by ELISA indicated maximum Apo2L concentrations of $3.8,32.1$, and $246 \mu \mathrm{g} / \mathrm{mL}$ at the $0.1,1$, and 
$10 \mathrm{mg} / \mathrm{kg}$ doses. On the basis of serum concentrations determined 5 minutes, 1 hour, and 8 hours after injection of $10 \mathrm{mg} / \mathrm{kg}$ Apo2L on day 7, we estimated the pharmacokinetic half-life of the protein in plasma to be $\sim 32$ minutes. We did not detect anti-Apo2L antibody titers in any of the treated animals (data not shown), suggesting that the soluble Apo2L protein was not highly immunogenic. Thus, exposure of cynomolgus monkeys to Apo2L at $0.1-10$ $\mathrm{mg} / \mathrm{kg} / \mathrm{d}$ over 7 days does not induce detectable toxicity; for comparison, TNF induced severe toxicity at 0.003 $\mathrm{mg} / \mathrm{kg} / \mathrm{d}$ (data not shown).

Antitumor activity of Apo2L in vitro. To explore the activity of Apo2L against a spectrum of cancer cell types, we tested its effect in vitro on the growth of 39 different cell lines derived from cancers of the colon, lung, breast, central nervous system, and kidney, and from malignant melanoma (Figure 3). Apo2L exerted a cytostatic effect on 16 of the cell lines, inhibiting cell growth by $50-100 \%$ compared with controls. Apo2L exerted a cytotoxic effect in 16 additional cell lines, reducing cell numbers by $50-100 \%$ compared with initial levels. Apo2L exhibited a similar scope of activity against cell lines derived from leukemia, prostate cancer, and ovarian cancer (data not shown). Thus, Apo2L inhibits proliferation or triggers cell death in vitro in a majority of cell lines derived from some of the most prevalent types of human cancer. There was no apparent correlation between sensitivity of the cell lines to Apo2L and other known biomarkers, including p53 status.

Antitumor activity of Apo2L in vivo. To investigate whether Apo2L has antitumor activity in vivo, we injected athymic nude mice subcutaneously with HCT116 human colon carcinoma cells, which showed intermediate sensitivity to Apo2L in vitro (Figure $3 \mathrm{a}$ ), and allowed tumors to establish over a 10-day period. We then gave the mice a single intratumoral (Figure $4 a$ ) or intraperitoneal (Figure 4, b and c) injection of Apo2L $(15 \mathrm{mg} / \mathrm{kg})$; 3 or 6 hours later, we examined the tumors for evidence of apoptosis. We detected Apo2L-dependent cleavage of the caspase-3 substrate poly-ADP ribose polymerase (PARP) by immunoblot analysis of tumor homogenates (Figure 4, a and b), which indicated apoptosis induction by Apo2L. In addition, we detected a substantial increase in the number of apoptotic cells in histological sections of tumors from mice treated with an intraperitoneal injection of Apo2L, as compared with vehicle (Figure 4, $c$ and $d$ ). Thus, systemic injection of Apo2L triggers apoptotic cell death in established tumor xenografts.

To test if Apo2L can affect the establishment of new tumors, we injected nude mice with HCT116 cells and, 1 day later, began treatment with intraperitoneal injections of vehicle or Apo2L (15 mg/kg/d) over 2 or 14 days; for comparison, we treated mice with the DNA-damaging agent 5-fluorouracil (5-FU) (25 mg/kg/d over 14 days), which is an established colon cancer chemotherapeutic drug (32) (Figure 5a). Apo2L reduced the incidence of tumors substantially: a 2-day treatment prevented tumor formation in $30 \%$ of the mice, and a 14-day treatment prevented tumor formation in $70 \%$ of the mice. In contrast, $95 \%$ of the 5 -FU-treated mice and $100 \%$ of controls developed tumors within 10-20 days. To investigate whether Apo2L and 5-FU could cooperate in blocking establishment of new tumors, we used a similar model, except that

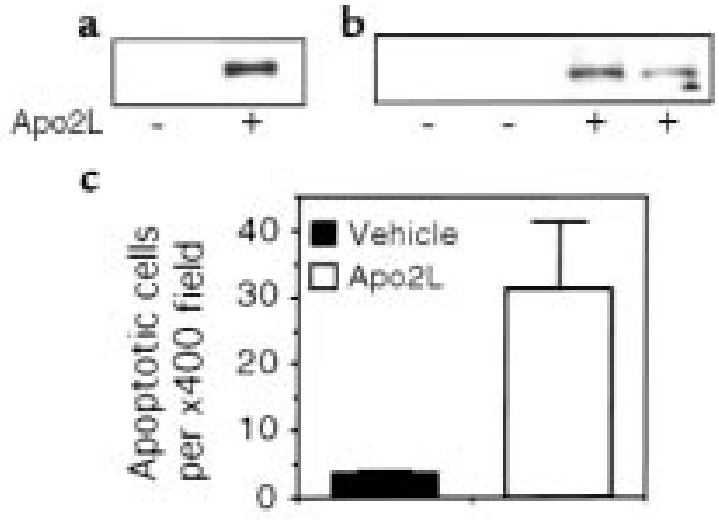

d

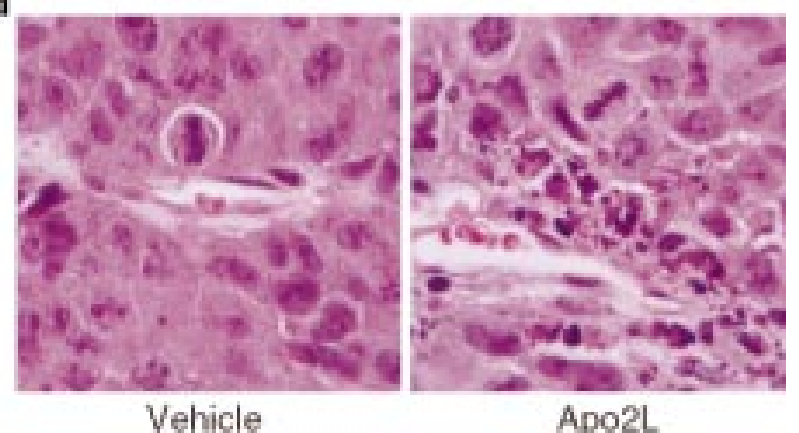

Figure 4

Induction of tumor cell apoptosis by Apo2L in vivo. Nude mice with subcutaneous HCT116 tumors were treated with vehicle (-) or Apo2L $(+)(15 \mathrm{mg} / \mathrm{kg})$ by intratumoral (a) or intraperitoneal (b-d) injection. After 3 hours (a) or 6 hours (b), tumor cell extracts were assayed for caspase- 3 activity by immunoblot analysis of the $85-\mathrm{kDa}$ cleavage product of PARP. (c) Histological sections of tumors from vehicle- or Apo2L-treated animals were stained with hematoxylin and eosin, and apoptotic figures were counted in 10 random $\times 400$ fields per tumor (mean \pm SD). (d) Hematoxylin and eosin-stained tumor sections from vehicle- or Apo2L-treated animals.
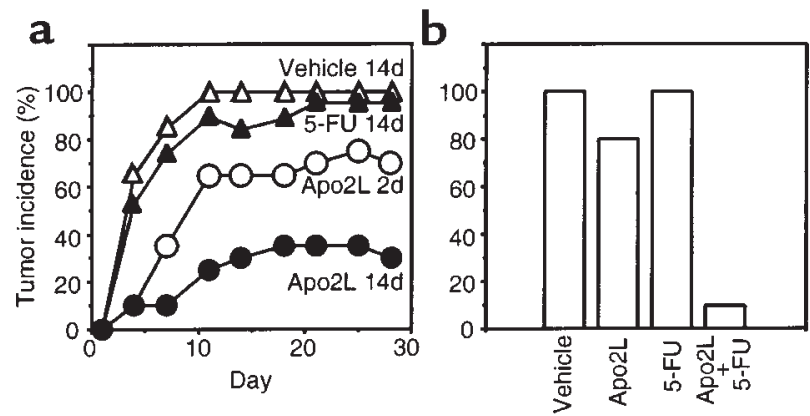

\section{Figure 5}

Effect of Apo2L on tumor formation. (a) Nude mice (10 per group) were injected subcutaneously with HCT116 cells; 1 day later, they were injected intraperitoneally with vehicle or Apo2L $(15 \mathrm{mg} / \mathrm{kg} / \mathrm{d})$ for 2 days or 14 days, or with 5 -FU $(25 \mathrm{mg} / \mathrm{kg} / \mathrm{d})$ for 14 days. The appearance of tumors was assessed every few days for a period of 4 weeks. (b) Nude mice (10 per group) were injected with HCT116 cells. On days 1 and 2 after inoculation, the mice received intraperitoneal injections of vehicle, Apo $2 \mathrm{~L}(0.5 \mathrm{mg} / \mathrm{kg} / \mathrm{d}), 5-\mathrm{FU}(5 \mathrm{mg} / \mathrm{kg} / \mathrm{d})$, or Apo2L plus 5-FU. The appearance of tumors of measurable size was assessed on day 15. 
we treated the animals over 2 days with significantly lower doses of Apo2L (0.5 mg/kg/d), 5-FU (5 mg/kg/d), or both molecules (Figure $5 \mathrm{~b}$ ). Apo2L prevented tumor formation in $20 \%$ of the animals, and 5-FU did not reduce tumor frequency; the 2 agents together blocked tumor establishment in $90 \%$ of the mice. Thus, in this model of de novo establishment of HCT116-derived tumors, Apo2L and 5-

$\mathbf{a}$

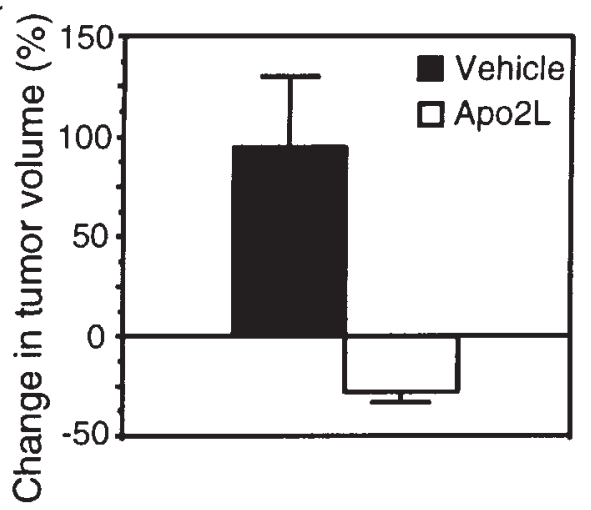

b

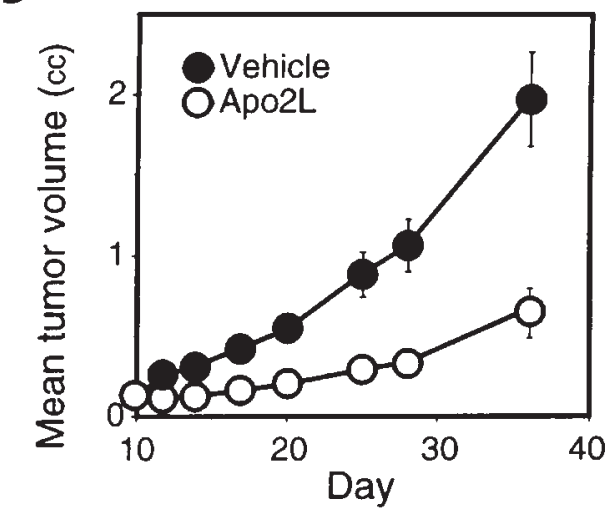

c

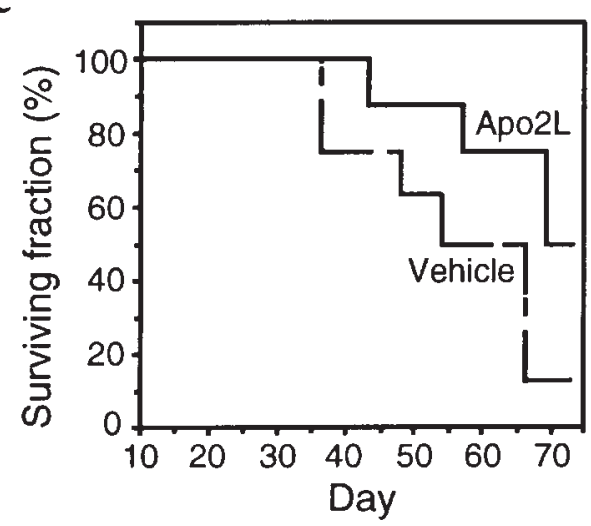

Figure 6

Effect of Apo2L on growth of established HCT116 tumors and on survival. (a) Nude mice carrying HCT116 tumors (5 per group) received vehicle or Apo $2 \mathrm{~L}(5 \mathrm{mg} / \mathrm{kg} / \mathrm{d}$ ) over 3 days through intraperitoneal infusion minipumps, and tumor volume was measured. (b) Nude mice carrying HCT116 tumors (10 per group) received intraperitoneal injections of vehicle or Apo2L (10 mg/kg/d) on days 10-14 and 16-20 after tumor cell inoculation, and tumor volume was measured (mean \pm SEM). (c) Independent groups of mice were treated as in $\mathbf{b}$ with vehicle (dashed line) or Apo2L (solid line), and were followed for survival.
FU cooperate effectively at preventing tumor growth.

Next, we tested the effect of Apo2L treatment on the progression of established tumors. We injected nude mice subcutaneously with HCT116 cells and allowed tumors to form and grow to a volume of $\sim 150 \mathrm{~mm}^{3}$. We then treated the mice intraperitoneally with vehicle or Apo2L (5 $\mathrm{mg} / \mathrm{kg} / \mathrm{d}$ ), delivered over 3 days using osmotic minipumps (Figure 6a). In vehicle-treated animals, the mean tumor volume nearly doubled during the 3-day experimental period; in contrast, in Apo2L-treated animals, the mean tumor volume decreased by $25 \%$. To study longer-term effects of Apo2L on HCT116 tumor growth, we carried out a similar experiment - except that we treated the animals intermittently with 2 courses of Apo2L (each at 10 $\mathrm{mg} / \mathrm{kg} / \mathrm{d}$ intraperitoneally over 5 days) - and followed tumor size over 4 weeks (Figure 6b). In vehicle-treated animals, the mean tumor volume increased to $2.0 \mathrm{cc}$, whereas in Apo2L-treated mice the mean tumor volume increased to $0.7 \mathrm{cc}$. Thus, treatment with Apo2L inhibited HCT 116 tumor growth by $65 \%$. In a similar study, we tested the effect of Apo2L on long-term survival of mice bearing HCT116 tumors (Figure 6c). Of the vehicle-treated animals, $87 \%$ died by day 66 after tumor cell injection; in contrast, $50 \%$ of the Apo2L-treated animals survived for at least 72 days. Hence, treatment of mice carrying HCT116 tumors with Apo2L can substantially attenuate tumor progression and improve survival.

To assess the in vivo activity of Apo2L on additional cancer cell lines, we injected nude mice subcutaneously with COLO201 or COLO205 human colon carcinoma cells, allowed solid tumors to establish, and gave the mice daily intraperitoneal injections of Apo2L. Over a treatment period of 4 days, the mean tumor volume of control animals increased by $225 \%$ (COLO201) or $375 \%$ (COLO205) (Figure 7, a and b). Apo2L caused a dosedependent suppression of tumor growth, reaching a maximal effect at $10-30 \mathrm{mg} / \mathrm{kg}$; COLO205 tumors in mice treated with the high Apo2L dose showed a $45 \%$ reduction in mean tumor volume (Figure $7 \mathrm{~b}$ ).

Next, we tested the activity of Apo2L and 5-FU against COLO205 tumors; in addition, we measured the combined effect of these 2 agents. We treated mice bearing subcutaneous tumors of $\sim 100 \mathrm{~mm}^{3}$ with two 5-day treatment courses of vehicle, Apo2L (30 mg/kg/d), 5-FU (25 $\mathrm{mg} / \mathrm{kg} / \mathrm{d}$ ), or Apo2L plus 5-FU, and followed tumor volume over 4 weeks (Figure 7c). In vehicle-treated animals, the mean tumor volume increased to $1.72 \mathrm{cc}$. Apo2L markedly suppressed tumor growth during the two 5-day treatment periods; tumor growth later resumed at a slower rate compared with controls, and the tumors reached a mean volume of $0.42 \mathrm{cc}$, indicating a $73 \%$ inhibition of tumor growth. In 5-FU-treated animals, mean tumor volume reached $0.75 \mathrm{cc}$, indicating a $56 \%$ inhibition of tumor growth compared with the control group. Combined treatment with Apo2L and 5-FU caused marked tumor regression during the treatment periods. Three out of 10 animals injected with Apo2L and 5-FU showed complete tumor elimination. The other 7 animals in this group showed little or no increase in mean tumor volume by day 28 , as compared with their initial tumor volume on day 5 . Histological sections of 1 of the cured animals confirmed the absence of tumor mass; the other 2 animals remained 
tumor free during a 90-day follow-up period.

We tested also the ability of Apo2L to cooperate with another established colon cancer chemotherapeutic drug, the topoisomerase inhibitor CPT-11 (33). In mice carrying subcutaneous COLO205 tumors of $\sim 120 \mathrm{~mm}^{3}$, Apo2L $(30 \mathrm{mg} / \mathrm{kg} / \mathrm{d}$ on days 5-9 and $12-16)$ or CPT-11 $(80 \mathrm{mg} / \mathrm{kg} / \mathrm{d}$ on days 5,9 , and 13 ) each caused substantial inhibition of tumor growth (Figure $7 \mathrm{~d}$ ). The combination of Apo2L plus CPT-11 caused tumor shrinkage within a few days, leading to complete tumor ablation in 8 out of 9 animals (89\%). Essentially identical results were obtained in repeated studies that assessed the combination of Apo2L with 5-FU or CPT-11 (not shown). Thus, Apo2L cooperates synergistically with 5-FU or CPT-11 to cause regression or complete remission of established tumors.

\section{Discussion}

Although TNF and FasL have potent cytotoxic activity against many types of tumor cells, the application of these death ligands to cancer therapy has been restricted by their severe toxicity to normal tissues. The discovery of Apo2L as a death ligand, with its wide tissue-mRNA distribution and its unique receptor system, suggested that this ligand might be more suitable than TNF or FasL for systemic cancer therapy.

Our initial goal toward preclinical assessment of Apo2L was to generate a recombinant molecule that forms stable, biologically active trimers, without fusion to foreign sequences. Previous work suggested that soluble versions of Apo2L require fusion to epitope tags and/or aggregation with antibodies directed to the tags for potent biological activity $(7,11)$. A recent report describes a biologically active fusion protein that contains Apo2L extracellular sequence (amino acids 95-281) linked to a trimerizing leucine zipper (LZ-TRAIL); this protein was expressed in Chinese hamster ovary cells (28). Our data show that active, homotrimeric soluble Apo2L (amino acids 114-281) can be made in bacteria without fusion to heterologous sequences; this recombinant protein is likely to be less immunogenic in humans than Apo2L fusion proteins and thus, perhaps, more suitable for repeated dosing. Consistent with this notion, 7 daily injections of soluble human Apo2L in nonhuman primates did not induce a detectable antibody response by the end of study on day 9 .

Given the severe systemic toxicity of TNF and FasL, our next goal was to test for any toxicity that might be associated with Apo2L injection. Much of TNF's toxicity is caused by activation of the proinflammatory transcription factor NF- $\kappa B(5)$. The NF- $\kappa B-i n d u c i n g$ activity of Apo2L in cultured vascular endothelial cells was markedly weaker than that of TNF, suggesting that Apo2L may not have significant proinflammatory activity. Furthermore, Apo2L was not cytotoxic toward cultured normal cells from endothelial, epithelial, fibroblastic, smooth muscle, or astrocytic origin, supporting the notion that this ligand is not generally cytotoxic to nontransformed cells. Walczak et al. reported resistance of several normal cell types to LZ-TRAIL, with the exception of cultured astrocytes (28).

We investigated also whether intravenous injection of

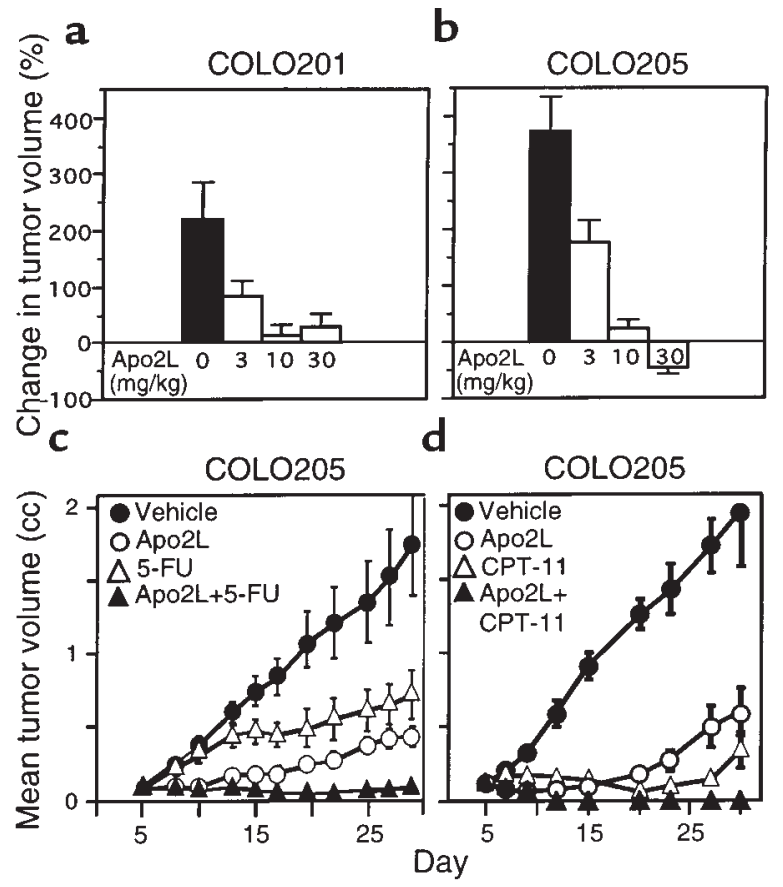

Figure 7

Effect of Apo2L on growth of established COLO205 tumors. (a and b) Nude mice carrying COLO201 (a) or COLO205 (b) tumors (10 per group) were injected intraperitoneally with vehicle or $A p o L L(3,10$, or 30 $\mathrm{mg} / \mathrm{kg} / \mathrm{d}$ ) over 4 days, and the tumor volume was measured. (c) Nude mice carrying COLO205 tumors (10 per group) received intraperitoneal injections of vehicle (closed circles) or Apo2L (30 mg/kg/d; open circles), 5 -FU ( $25 \mathrm{mg} / \mathrm{kg} / \mathrm{d}$; open triangles), or Apo2L plus 5-FU (closed triangles) on days 5-9 and 12-16, and tumor volume was determined. (d) Nude mice carrying COLO205 tumors ( 9 per group) received intraperitoneal injections of vehicle (closed circles) or Apo $2 \mathrm{~L}(30 \mathrm{mg} / \mathrm{kg} / \mathrm{d}$; open circles) on days 5-9 and 12-16, or CPT-11 ( $80 \mathrm{mg} / \mathrm{kg} / \mathrm{d}$; open triangles) on days 5, 9, and 13, or Apo2L plus CPT-11 (closed triangles), and tumor volume was determined. The data are mean \pm SEM.

Apo2L causes toxicity in nonhuman primates. Repeated systemic exposure of cynomolgus monkeys to significant doses of Apo2L did not cause detectable changes in an extensive set of clinical and histopathological tests, suggesting that the administration of this protein is remarkably safe. These results are in agreement with recent findings in mice (28). Given that rodents are much less sensitive to the toxic effects of TNF than are humans (31), the apparent safety of Apo2L in primates is a key advance toward the testing of this molecule in humans.

Our third goal was to assess the antitumor activity of Apo2L in models of cancer. A broad spectrum of cancer cell lines, including many that have p53 mutations, exhibited sensitivity in vitro to Apo2L. This finding is consistent with previous reports that Apo2L is cytotoxic toward cell lines from glioma (34), melanoma (35), and Kaposi's sarcoma (36), and against cells of certain hematological malignancies (37). Our studies in colon tumor xenograft models indicate that Apo2L can inhibit establishment de novo of tumors. In mice bearing sizable subcutaneous tumors, Apo2L initiated tumor cell apoptosis, consistent with its apoptosis-inducing activity in vitro. Exposure to Apo2L caused tumor regression 
or suppressed tumor growth, which resulted in longterm inhibition of tumor progression. These results agree with the findings of Walczak et al., who studied mainly a xenograft model based on the MDA-MB-231 breast carcinoma cell line (28).

We tested Apo2L's ability to cooperate with established chemotherapeutic drugs to exert antitumor activity. Apo2L acted synergistically with 5-FU, and particularly with CPT-11, to cause either tumor regression or complete tumor remission. Perhaps activation of distinct apoptosis-signaling mechanisms by the death ligand and the DNA-damaging agents (3) contributes to their cooperation. In addition, 5-FU can upregulate mRNA expression of DR5 (12), which may augment apoptosis induction by Apo2L.

In conclusion, we have generated a potently active recombinant soluble human Apo2L molecule that is devoid of exogenous sequences. Repeated administration of this molecule to nonhuman primates appeared remarkably safe and nonimmunogenic. In vitro, soluble Apo2L exerted cytostatic or cytotoxic effects on a wide variety of tumor cell lines, but not on several types of normal cells. In vivo, soluble Apo2L showed substantial antitumor activity in xenograft models based on several colon carcinoma cell lines, and exhibited synergistic activity with 5-FU or CPT-11, causing marked regression or complete remission of tumors. Thus, Apo2L may prove to be a useful new tool to fight cancer cells, leaving normal cells unharmed.

\section{Acknowledgments}

We thank Ed Sausville and Jill Johnson of the National Cancer Institute for testing of Apo2L with the NCI tumor cell line panel; Chris Clark for comments on the manuscript; Anan Chuntharapai and Jin Kim for antiApo2L antibodies; Glynis MacCray for help with cell culture; and Debbie Thomas, Mary Napier, Kip Benyunes, Robert Cohen, and Vishva Dixit for advice.

1. Jacobson, M.D., Weil, M., and Raff, M.C. 1997. Programmed cell death in animal development. Cell. 88:347-354.

2. Nagata, S. 1997. Apoptosis by death factor. Cell. 88:355-365.

3. Ashkenazi, A., and Dixit, V.M. 1998. Death receptors: signaling and modulation. Science. 281:1305-1308

4. Levine, A.J. 1997. p53, the cellular gatekeeper for growth and division. Cell. 88:323-331.

5. Tartaglia, L., and Goeddel, D. 1992. Two TNF receptors. Immunol. Today. 13:151-153.

6. Pitti, R.M., et al. 1996. Induction of apoptosis by Apo-2 ligand, a new member of the tumor necrosis factor receptor family. J. Biol. Chem. 271:12687-12690.

7. Wiley, S.R. et al. 1995. Identification and characterization of a new member of the TNF family that induces apoptosis. Immunity. 3:673-682.

8. Pan, G., et al. 1997. The receptor for the cytotoxic ligand TRAIL. Science. 276:111-113.

9. Sheridan, J.P., et al. 1997. Control of TRAIL-induced apoptosis by a family of signaling and decoy receptors. Science. 277:818-821.

10. Pan, G., et al. 1997. An antagonist decoy receptor and a new death domaincontaining receptor for TRAIL. Science. 277:815-818.
11. Walczak, H., et al. 1997. TRAIL-R2: a novel apoptosis-mediating receptor for TRAIL. EMBO J. 16:5386-5397.

12. Wu, G.S., et al. 1997. KILLER/DR5 is a DNA damage-inducible p53-regulated death receptor gene. Nat. Genet. 17:141-143.

13. Schneider, P., et al. 1997. Characterization of two receptors for TRAIL. FEBS Lett. 416:329-334.

14. Chaudhary, P.M., et al. 1997. Death receptor 5, a new member of the TNFR family, and DR4 induce FADD-dependent apoptosis and activate the NF$\mathrm{kB}$ pathway. Immunity. 7:821-830.

15. Screaton, G.R., et al. 1997. TRICK2, a new alternatively spliced receptor that transduces the cytotoxic signal from TRAIL. Curr. Biol. 7:693-696.

16. Degli-Esposti, M., et al. 1997. Cloning and characterization of TRAIL-R3, a novel member of the emerging TRAIL receptor family. J. Exp. Med. 186:1165-1170

17. Mongkolsapaya, J., et al. 1998. Lymphocyte inhibitor of TRAIL: a new receptor protecting lymphocytes from the death ligand TRAIL. J. Immunol. 160:3-6.

18. Marsters, S.A., et al. 1997. A novel receptor for Apo2L/TRAIL contains a truncated death domain. Curr. Biol. 7:1003-1006.

19. Degli-Esposti, M.A., et al. 1997. The novel receptor TRAIL-R4 induces NF$\mathrm{kB}$ and protects against TRAIL-mediated apoptosis, yet retains an incomplete death domain. Immunity. 7:813-820.

20. Pan, G., Ni, J., Yu, G.L., Wei, Y.F., and Dixit, V.M. 1998. TRUNDD, a new member of the TRAIL receptor family that antagonizes TRAIL signaling. FEBS Lett. 424:41-45.

21. Emery, J.G., et al. 1998. Osteoprotegerin is a receptor for the cytotoxic ligand TRAIL. J. Biol. Chem. 273:14363-14367.

22. Simonet, W.S., et al. 1997. Osteoprotegerin: a novel secreted protein involved in the regulation of bone density. Cell. 89:309-319.

23. Gruss, H.J., and Dower, S.K. 1995. Tumor necrosis factor ligand superfamily: involvement in the pathology of malignant lymphomas. Blood. 85:3378-3404.

24. Tanaka, M., Itai, T., Adachi, M., and Nagata, S. 1998. Downregulation of Fas ligand by shedding. Nat. Med. 4:31-36.

25. Mariani, S.M., Matiba, B., Armandola, E.A., and Krammer, P.H. 1997. Interleukin 1 beta-converting enzyme related proteases/caspases are involved in TRAIL-induced apoptosis of myeloma and leukemia cells. J. Cell Biol. 137:221-229.

26. Mariani, S., and Krammer, P. 1998. Surface expression of TRAIL/Apo2 ligand in activated mouse T and B cells. Eur. J. Immunol. 28:1492-1498.

27. Marsters, S., et al. 1996. Activation of apoptosis by Apo-2 ligand is independent of FADD but blocked by CrmA. Curr. Biol. 6:750-752.

28. Walczak, H., et al. 1999. Tumoricidal activity of tumor necrosis factorrelated apoptosis-inducing ligand in vivo. Nat. Med. 5:157-163.

29. Scholtissek, S., and Grosse, F. 1988. A plasmid vector system for the expression of a triprotein consisting of beta-galactosidase, a collagenase recognition site and a foreign gene product. Gene. 62:55-64.

30. Christian, M., et al. 1997. Promising new agents under development by the Division of Cancer Treatment, Diagnosis, and Centers of the National Cancer Institute. Semin. Oncol. 24:219-240.

31. Vassalli, P. 1992. The pathophysiology of tumor necrosis factors. Annu. Rev. Immunol. 10:411-452.

32. Leichman, C., et al. 1995. Phase II study of fluorouracil and its modulation in advanced colorectal cancer: a southwest oncology group study. J. Clin. Oncol. 13:1303-1311.

33. Vanhoefer, U., et al. 1999. Phase I study of a weekly schedule of irinotecan, high-dose leucovorin, and infusional fluorouracil as first-line chemotherapy in patients with advanced colorectal cancer. J. Clin. Oncol. 17:907-913.

34. Rieger, J., Naumann, U., Glaser, T., Ashkenazi, A., and Weller, M. 1998. Apo2 ligand: a novel lethal weapon against malignant glioma? FEBS Lett. 427:124-128.

35. Thomas, W.D., and Hersey, P. 1998. TNF-related apoptosis-inducing ligand (TRAIL) induces apoptosis in Fas ligand-resistant melanoma cells and mediates CD4 T cell killing of target cells. J. Immunol. 161:2195-2200.

36. Mori, S., Murakami-Mori, K., Nakamura, S., Ashkenazi, A., and Bonavida, B. 1999. Sensitization of AIDS-Kaposi's sarcoma cells to Apo2 ligandinduced apoptosis by actinomycin D. J. Immunol. 162:5616-5623.

37. Snell, V., et al. 1997. Activity of TNF-related apoptosis-inducing ligand (TRAIL) in haematological malignancies. Br. J. Haematol. 99:618-624.

38. Marsters, S., et al. 1996. Apo3, a new member of the tumor necrosis factor receptor family, contains a death domain and activates apoptosis and NFкB. Curr. Biol. 6:1669-1676. 